\title{
Dielectric water sorption analysis
}

\author{
M. Giacomelli Penon and S. J. Picken \\ Department NanoStructured Materials, Faculty of Applied Sciences, Delft University of Technology, \\ Julianalaan 136, 2628 BL Delft, The Netherlands \\ M. Wübbenhorst \\ Acoustics and Thermal Physics Section, KU Leuven, Celestijnenlaan 200D, BE-3001 Heverlee, Belgium \\ G. de Vos and J. van Turnhout \\ Department of NanoStructured Materials, Faculty of Applied Sciences, Delft University of Technology, \\ Julianalaan 136, 2628 BL Delft, The Netherlands
}

(Received 25 July 2006; accepted 30 September 2006; published online 28 November 2006)

\begin{abstract}
The sorption of water vapor in Kapton ${ }^{\circledR}$ (polyimide) films has been investigated with a new method, dielectric sorption analysis (DSA). The technique is based on high-resolution time-resolved capacitance measurements performed during exposure of an organic coating to humidified nitrogen. The basis of the method is described together with experimental details related to the capacitance measurement, long-term stability, reproducibility, and other characteristics of the setup. The technique allows the determination of the sorption/desorption and diffusion characteristics of a coating on a time scale varying from seconds to days. Mass transport properties derived from complementary weight measurements are in good agreement with the DSA results. Real-time monitoring of the moisture sorption in organic coatings demonstrates the potential of the technique in the field of nondestructive coating inspection and testing. (C) 2006 American Institute of Physics. [DOI: 10.1063/1.2370739]
\end{abstract}

\section{INTRODUCTION}

Mass transport properties of organic coatings are important for several applications, such as food packaging, beverage cans, protective paints, or composite materials. ${ }^{1-3} \mathrm{~A}$ measure for the barrier or permeation behavior of a coating is the time lag, i.e., the diffusion time for gases or liquids to propagate through a film of a given thickness $d,{ }^{4}$ which depends on the solubility $S$ (sorption) and the diffusion constant $D$. These two material properties depend on both the coating material and the permeant (e.g., methane, benzene, gasoline, oil, etc). ${ }^{5}$

The techniques used most frequently to investigate sorption and diffusion properties are isothermal weight measurements, thermogravimetric analysis (TGA), and permeability $(P=D S)$ measurements. For the latter, typically two-chamber cells are used, which are separated by the membrane to be tested. Monitoring the time evolution of the gas flow or the pressure difference allows the determination of the dynamic $(D)$ and static $(S, P)$ transport properties. A good review of the current state-of-the-art techniques is given by Rabek. ${ }^{6}$ Gravimetric measurements are the most straightforward methods to determine the sorption behavior of coatings. The mass increase is monitored while the coating is exposed to the gas/liquid of interest under isothermal conditions.

The main disadvantage of the above-mentioned techniques is the inability to monitor the very first seconds of the dynamic transport characteristics. The inability to use a substrate is another disadvantage for some of the techniques. This makes it difficult to characterize paints or samples of which the top micron layer has been chemically or physically modified, e.g., by weathering or oxidation.
Therefore we propose an alternative approach that aims to measure water sorption with a high sensitivity and with a fast acquisition rate using a capacitive measurement. ${ }^{7}$ The high sensitivity stems from the fact that water has a large dipole moment and high relative dielectric constant ${ }^{8}$ causing a significant change of the dielectric constant at low moisture levels, i.e., when water starts to penetrate into the organic coating. The basic principles of the technique are described and validated by measuring the water vapor sorption of a Kapton ${ }^{\circledR}$ polyimide coating. It will become clear that measurements can be done over both short and long times. The former provides a particular advantage if the transport phenomena in surface layers are to be studied.

\section{EXPERIMENT}

\section{A. Materials}

To test our dielectric sorption setup we have used polyimide films (Kapton ${ }^{\circledR}$, DuPont) with a thickness of $50 \mu \mathrm{m}$. These films have a uniform thickness and their properties are stable. The chemical structure is shown in Fig. 1.

\section{B. Experimental setup}

The dielectric sorption technique is schematically shown in Fig. 2 and comprises the following components: a humidifier, a two-way gas switch, the measurement cell, and a dielectric analyzer. Gaseous nitrogen, originating from a liquid nitrogen source, is used as the working gas with a controlled temperature and flow rate. The dry gas is either moisturized in a humidifier (water flask+ buffer reservoir) or bypassed to the sample cell using two fast electromechanical two-way 


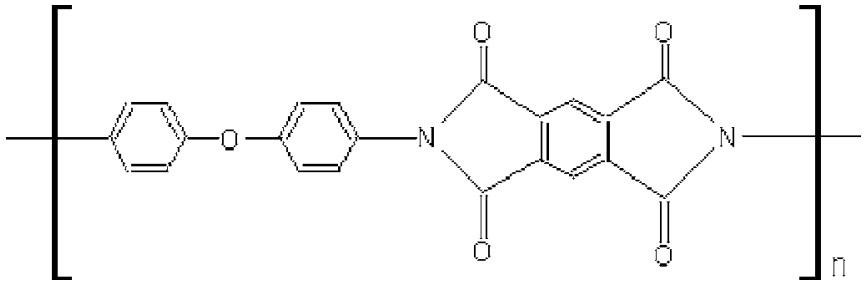

FIG. 1. Chemical structure of Kapton®.

valves (type Alcon $220 \mathrm{~V}$ ). The measuring cell consists of a closed compartment, in which the organic film is sandwiched between a porous top electrode and a solid bottom electrode, which are connected to a dielectric analyzer (Andeen Hagerling high precision $1 \mathrm{kHz}$ bridge, type 2500A). The gas inlets for dry and moisturized nitrogen are located close to the "quasifree" sample surface to ensure an effective and fast gas exchange.

Several measures have been taken to ensure mechanical stability and reliable electrical contacts. Furthermore water absorption on tube and cell surfaces is avoided in order to obtain good reproducibility. Teflon tubes have been used in the entire flow circuit, the sample cell is made from a low permittivity based polymer (Delrin ${ }$, DuPont), and all electrodes are coated with a gold layer. For the porous top electrode, a crucial component, a sheet of perforated steel of $100 \mu \mathrm{m}$ thick was used with holes of $100 \mu \mathrm{m}$ diameter at an average spacing of $115 \mu \mathrm{m}$ (see Fig. 3).

To ensure good contact between the porous top electrode and the coating, a constant load is applied with a weight with a value that depends on the type of coating. For Kapton ${ }^{\circledR}$ films a constant stress of about $20 \mathrm{kPa}$ is the maximum. Higher stresses result in plastic deformation causing a permanent imprint of the top electrode in the film. Lower stress values may give rise to poor contact of the electrodes with the film and an air gap will remain.

The bottom surface of the Kapton ${ }^{\circledR}$ film was coated with gold by vapor deposition to eliminate the influence of the air gap between the film and bottom electrode. The gold layer also blocks the uptake of water at the bottom surface.

Due to the small gas exchange volume $(\sim 1 \mathrm{ml})$ and a
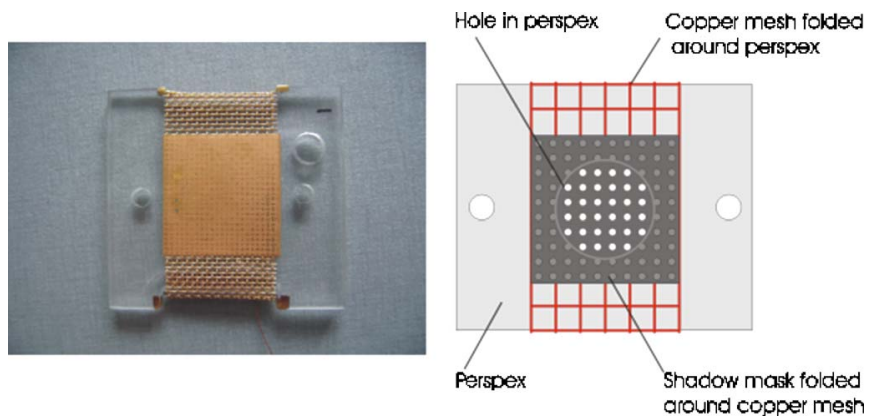

FIG. 3. Photograph and schematic representation of the porous screen used as top electrode in the DSA setup.

nitrogen flow of up to $7 \mathrm{ml} / \mathrm{s}$, a refreshment rate of about five times per second was realized.

The setup is placed in a temperature conditioned room at about $24.5^{\circ} \mathrm{C}\left( \pm 1^{\circ} \mathrm{C}\right)$ ambient temperature.

\section{Gravimetric experiments}

For the validation of the dielectric sorption analysis (DSA) results, gravimetric measurements were performed. The Kapton ${ }^{\circledR}$ samples were dried in dry nitrogen at room temperature (RT) for 5 days and then weighed on a precision balance. Subsequently, the sample was stored in a flow cell that was continuously flushed with moisturized nitrogen at $88 \%$ relative humidity $(\mathrm{RH})$ (at RT). The mass was measured by taking the sample out for a few seconds every $15 \mathrm{~min}$ (first hour), $20 \mathrm{~min}$ (second and third hour), and finally every 30 min until saturation of the sample mass was observed.

\section{Humidity measurement}

The humidity and the temperature of the dry and humidified nitrogen were monitored real time with a humidity meter (Testo 177-H1). This real-time measurement was achieved by splitting the gas inlets just before they enter the measurement cell and not in the cell itself as otherwise the humidity meter would have disturbed the sensitive dielectric measurements.

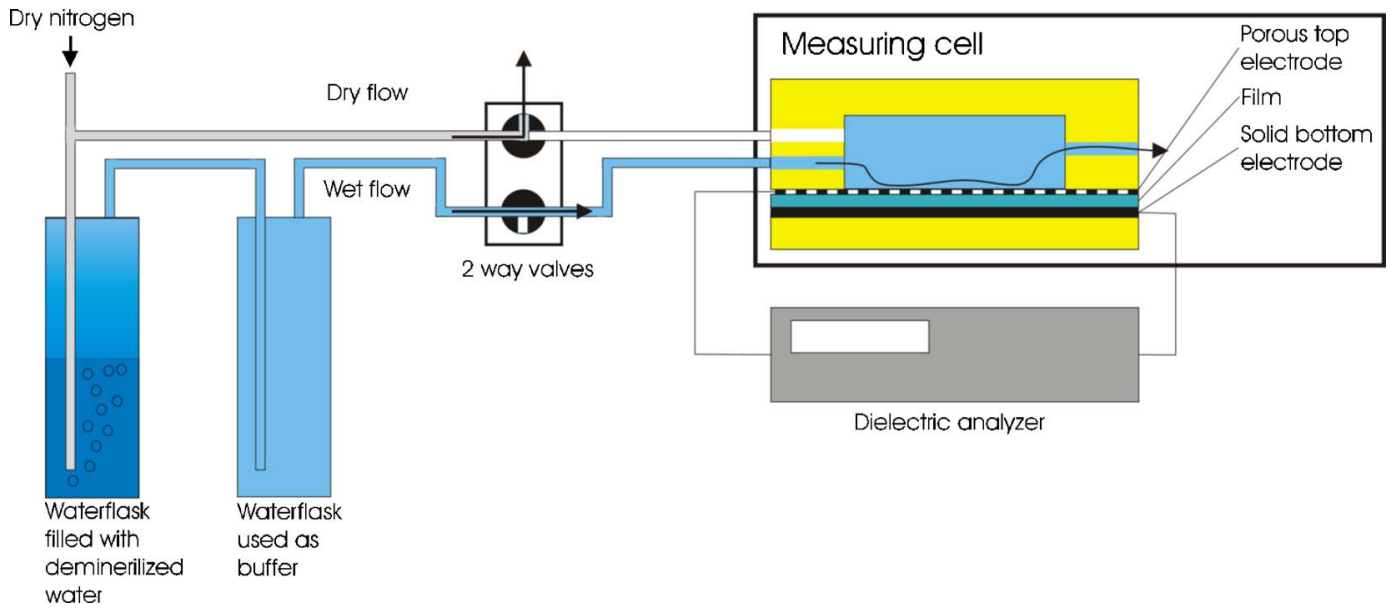

FIG. 2. Schematic drawing of the DSA setup. 

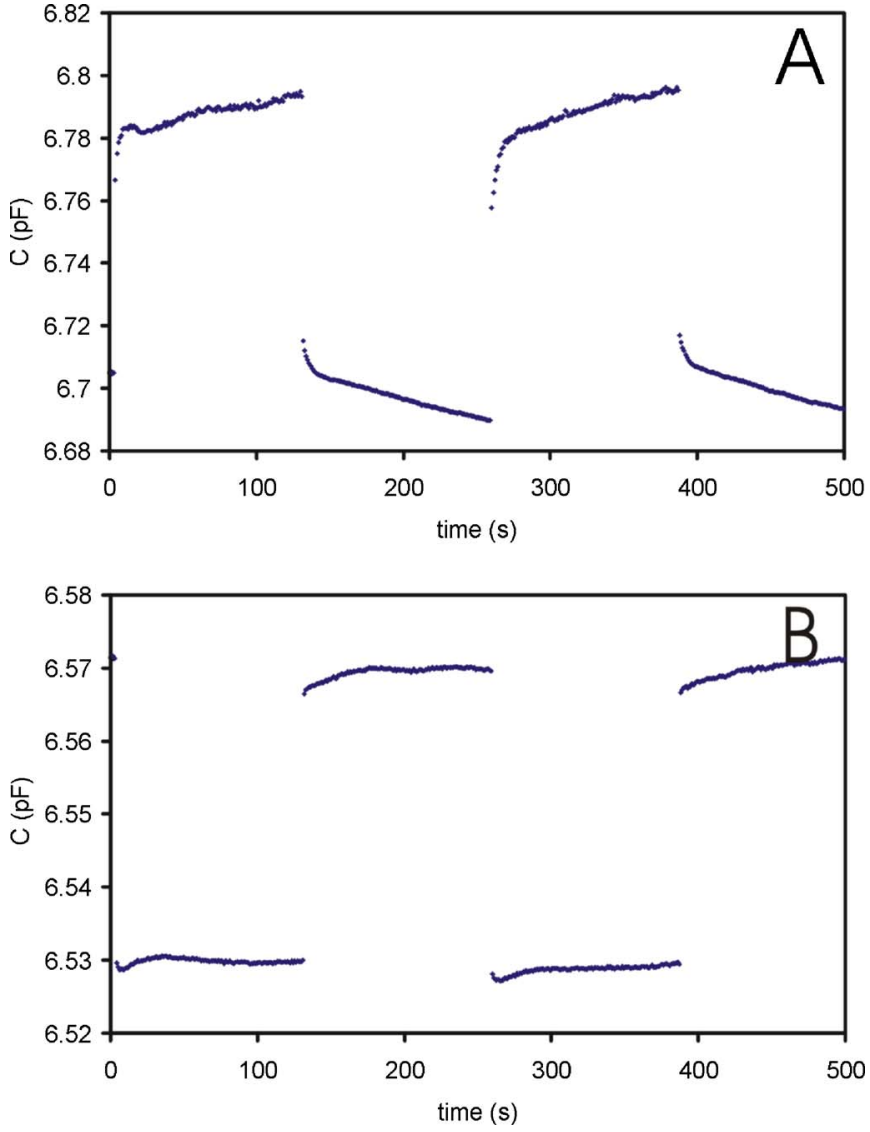

FIG. 4. (a) Generation of a block wave between the empty electrode system at room temperature. (b) Generation of a block wave between the empty electrode system at $60{ }^{\circ} \mathrm{C}$.

\section{RESULTS}

\section{A. Validation of switching behavior and stability 1. Conditions}

The temperature and the gas flow rate of the dry and humidified nitrogen were monitored real time. The temperature varied about $27^{\circ} \mathrm{C}\left(+/-1{ }^{\circ} \mathrm{C}\right)$, while the flow rate stayed constant. The humidity of the wet nitrogen flow was stable at $86 \% \mathrm{RH}(+/-1 \% \mathrm{RH})$.

\section{Response time}

The response time of the dielectric bridge (Andeen Hagerling high precision $1 \mathrm{kHz}$ bridge, type 2500A) was about $1 \mathrm{~s}$; this was checked by rapidly removing a glass fiber from between two brass electrodes, which gave a sharp stepwise change in the measured capacity.

To test whether a sharp square humidity profile wave was generated, when switching between dry and wet nitrogen, an empty electrode system was used.

As shown in Fig. 4(a), at room temperature a sharp square wave is not observed when switching from dry to wet nitrogen. However, when the electrodes were heated to $60^{\circ} \mathrm{C}$ a nice square wave was found [Fig. 4(b)]. We believe that at room temperature a thin water layer is formed on the electrodes, ${ }^{9}$ while at $60{ }^{\circ} \mathrm{C}$ this layer is not present on the electrodes. A water layer cannot be formed on the electrodes, if a sample is placed between them.

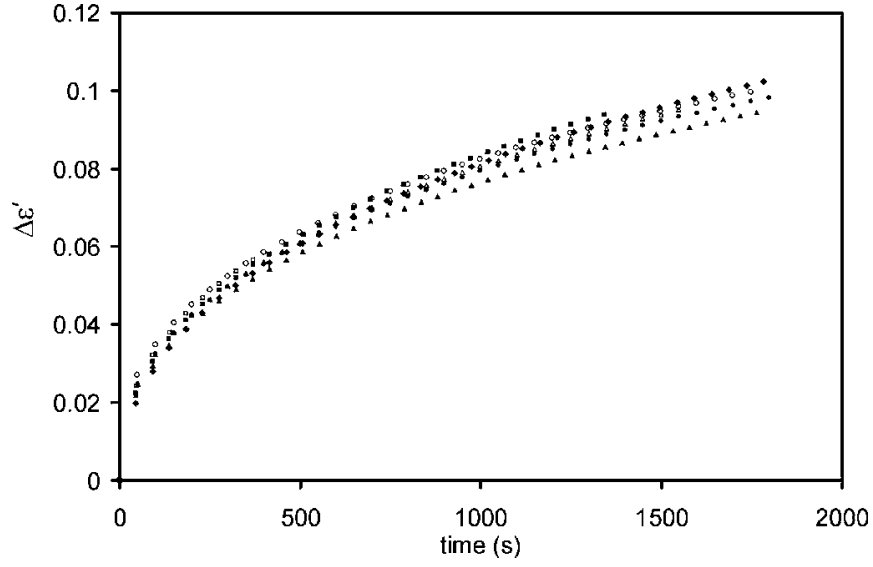

FIG. 5. Increase in dielectric permittivity with time of Kapton ${ }^{\circledR}$ for short times at $86 \% \mathrm{RH}$ and room temperature, repeated seven times.

In conclusion the measured response time of the dielectric analyzer was found to be in good agreement with the gas exchange rate (seven times a second).

\section{Stability}

The stability of the measurement cell was monitored using a piezoelectric force transducer. The transducer was placed on top of the cell, and directly after inserting a Kapton ${ }^{\circledR}$ film in the setup, a decrease of load was registered. This decrease was due to the settling of the film between the two electrodes and thermal and mechanical adjustments of the device as a whole. Therefore before actually starting the measurement, dry nitrogen was passed through the DSA setup for about $4 \mathrm{~h}$.

\section{Reproducibility}

After verification and characterizing the "empty" DSA equipment, a Kapton ${ }^{\circledR}$ film was repeatedly measured (seven times) for about $1800 \mathrm{~S}$. The results are shown in Fig. 5. After each measurement the Kapton ${ }^{\circledR}$ film was kept under ambient conditions for 1 day before starting the next measurement; therefore the coating initially was not entirely dry.

These results indicate that it is possible to obtain good and reproducible sample handling with the DSA. In addition the sample response showed that no chemical or physical changes occurred during the seven measurements.

To analyze the capacitive response, we use the following expression:

$$
\varepsilon^{\prime}=\frac{C, d}{\varepsilon_{0} A},
$$

where $\varepsilon^{\prime}$ is the dielectric permittivity of the sample, $C^{\prime}$ is the real part of the capacitance [F], $d$ the thickness of the sample $[\mathrm{m}], \varepsilon_{0}$ the permittivity of vacuum $\left(\mathrm{J}^{-1} \mathrm{C}^{2} \mathrm{~m}^{-1}\right)$, and $A$ the measured Kapton ${ }^{\circledR}$ area $\left(\mathrm{m}^{2}\right)$.

Sorption of water in the coating causes swelling of the coating. ${ }^{10}$ The resulting increase in $d$ gives a decrease in the capacitance [see Eq. (1)]. But in view of the small water uptake of Kapton ${ }^{\circledR}(\sim 2 \%$ in our case) this decrease is negligible in comparison with the increase in $\varepsilon^{\prime}$ as caused by the high dielectric constant of the penetrating water. 

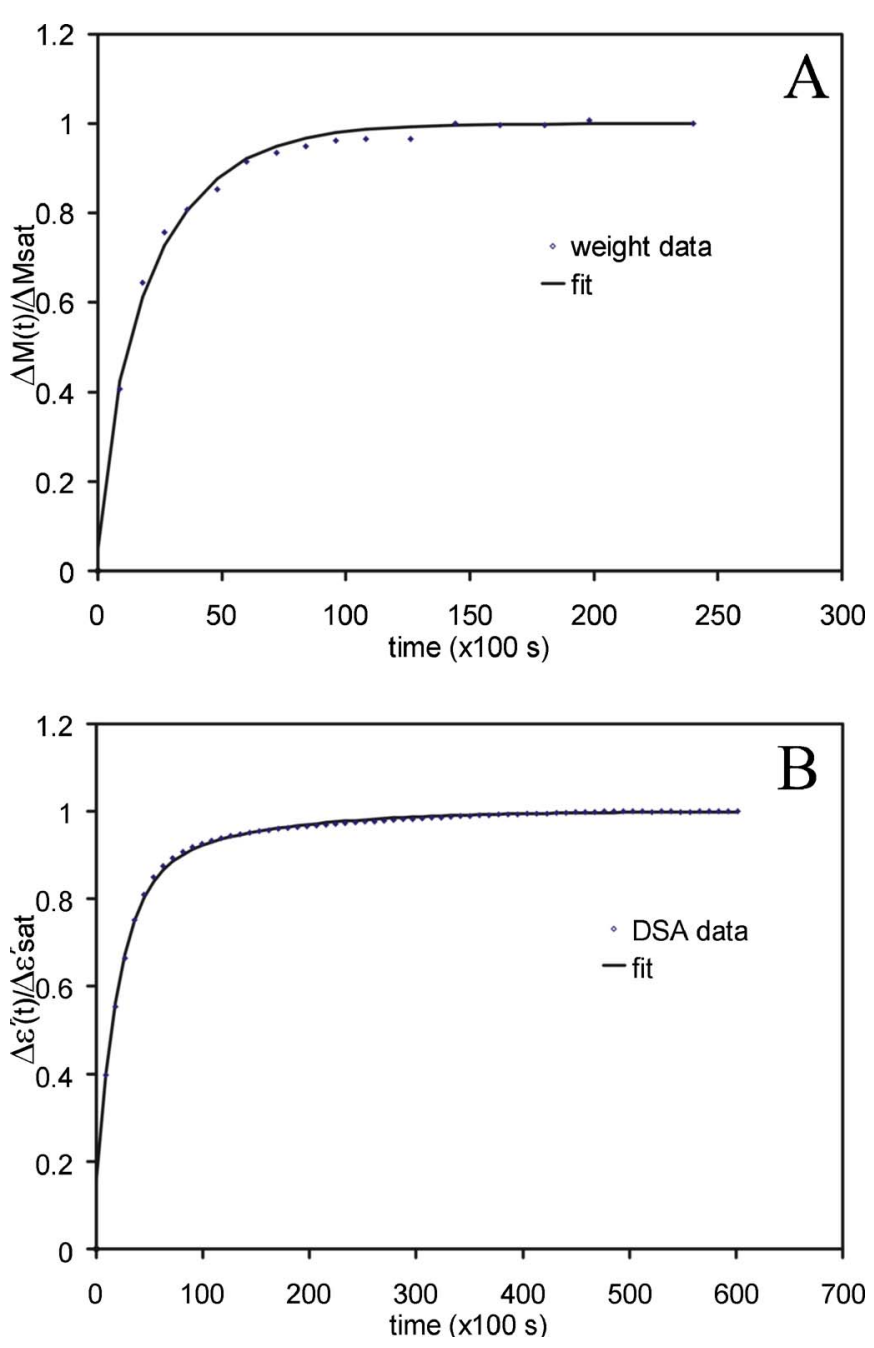

FIG. 6. (a) Normalized weight increase of fitted Kapton® at $86 \%$ RH and room temperature. The solid line represents the fit to the data using Eq. (2). (b) Normalized DSA data for Kapton $®$ at $86 \% \mathrm{RH}$ and room temperature. The solid line represents the fit with Eq. (2).

Since we are interested in changes of the dielectric constant as the result of water absorption it is useful to present the difference $\varepsilon^{\prime}(t)-\varepsilon^{\prime}(t=0)$, where $\varepsilon^{\prime}(t=0)$ is the initial value of $\varepsilon^{\prime}$ in the dry state at the start of the measurement, as shown in Fig. 5.

\section{B. Comparison between DSA results and gravimetric measurements}

Now a direct comparison between the DSA response and the gravimetric data of the Kapton ${ }^{\circledR}$ samples can be made The results of the gravimetric measurements are displayed in Fig. 6(a). The mass increase levels off, when the saturation concentration is reached. The data in Fig. 6(a) have been normalized by showing $\Delta M_{t} / \Delta M_{\mathrm{sat}}$. The DSA results are displayed in Fig. 6(b), where similar normalization has been applied to the capacitive response $\left(\Delta \varepsilon_{t}^{\prime} \Delta \varepsilon_{\mathrm{sat}}^{\prime}\right)$.

To relate the dielectric response to the actual mass increase, which is assumed to be solely due to the water uptake, we have to model the dielectric response and the mass response. For this a two-stage sorption mode ${ }^{11}$ with a relaxation term is used, ${ }^{12}$ shown in Eq. (2). The first term in Eq. (2) represents the "perfect" Fickian diffusion of water into a sample and the second term is the additional gradual uptake brought about by the long-term relaxation of the sample due to structural rearrangements induced by the diffusing medium, in this case water.

$$
\begin{aligned}
\frac{\Delta M_{t}}{\Delta M_{\mathrm{sat}}}= & \Phi\left\{1-\frac{8}{\pi^{2}} \sum_{n=0}^{\infty} \frac{1}{(2 n+1)^{2}} \exp [-(2 n\right. \\
& \left.\left.\left.+1^{2}\right) \frac{\pi^{2} D t}{4 d^{2}}\right]\right\}+(1-\Phi)[1-\exp (-k t)],
\end{aligned}
$$

where $M_{t}$ is the weight increase in time, $M_{\text {sat }}$ the weight increase at saturation, $d$ the half thickness [m], $D$ the diffusion coefficient $\left(\mathrm{m}^{2} \mathrm{~s}^{-1}\right), t$ the time $[\mathrm{s}], \Phi$ the equilibrium ratio constant which represents the ratio of the equilibria of the first stage to that of the second stage in the sorption, and $k$ the rate constant of the relaxation process $\left(\mathrm{s}^{-1}\right)$.

Note that the exponential series in Eq. (2) converges quickly, and using the first three terms therefore suffices. Crank $^{11}$ and Talbot ${ }^{17}$ derived an approximation to the inverse of the exponential part in Eq. (2). This greatly simplifies the calculation of $D$, (cf. Appendix A).

Fitting Eq. (2) to the weight results gives a diffusion coefficient of $4.8 \times 10^{-13} \mathrm{~m}^{2} \mathrm{~s}^{-1}$ [according to the literature $D$ varies between $(2-4) \times 10^{-13} \mathrm{~m}^{2} \mathrm{~s}^{-1}$ at $50{ }^{\circ} \mathrm{C}$ and $80 \%$ RH (Ref. 13)]. Already Hayward et al. ${ }^{19}$ have assumed that $\Delta \varepsilon_{t}^{\prime}$ is linearly dependent on $M_{t}$ and $\Delta \varepsilon_{\text {sat }}^{\prime}$ on $M_{\text {sat }}$ for their dielectric measurements (the validity of these assumptions is addressed in Appendix B). Using this assumption a diffusion coefficient of $5.0 \times 10^{-13} \mathrm{~m}^{2} \mathrm{~s}^{-1}$ is obtained for the DSA measurements. The resulting fit curves are shown in Figs. 6(a) and 6(b). This indicates that there is excellent agreement between the DSA results and the mass uptake curve.

The weight percentage of the saturation water content can be calculated from the mass measurements by $M_{\mathrm{sat}} / M_{\mathrm{dry}}$, resulting in $1.6 \% \mathrm{w} / \mathrm{w}$ (at RT and $86 \% \mathrm{RH}$ ). For DSA a dielectric mixing rule is necessary to account for the heterogeneous nature of a dielectric material containing a dispersed phase of a second component, in this case water. ${ }^{14,17}$ Lindqvist ${ }^{18}$ compared five formulas of mixing on various coatings for impedance measurements. We use a model, which assumes that the water (molecule) is dispersed in the matrix as spherical (or randomly dispersed ellipsoidal) clusters, resulting in the following relationship known as the Landau-Lifschitz-Looyenga (LLL) relation: ${ }^{15,16}$

$$
\varepsilon_{t}^{\prime 1 / 3}=\nu_{w} \varepsilon_{w}^{\prime 1 / 3}+\left(1-\nu_{w}\right) \varepsilon_{d}^{\prime 1 / 3},
$$

where $\varepsilon_{t}^{\prime}$ is the total dielectric permittivity of the saturated signal (water+Kapton ${ }^{\circledR}$, which is 2.593), $\varepsilon_{w}^{\prime}$ the dielectric permittivity of water (assumed to be 80 ), $\varepsilon_{d}^{\prime}$ is the dielectric permittivity of dry Kapton ${ }^{\circledR}$ (derived from measurements, 2.238), and $v_{w}$ the volume fraction of water at saturation. The volume percentage of water in Kapton ${ }^{\circledR}$ found using this model is $2.2 \% \mathrm{v} / \mathrm{v}$ (at RT and $86 \% \mathrm{RH}$ ). This corresponds to a weight percentage of $1.56 \% \mathrm{w} / \mathrm{w}$, since the specific density of Kapton ${ }^{\circledR}$ is $1.42 \mathrm{~g} / \mathrm{ml}$.

The results of $1.6 \% \mathrm{w} / \mathrm{w}$ and $1.56 \% \mathrm{w} / \mathrm{w}$ indicate a good agreement between the direct (gravimetric) and the indirect 
(DSA) approaches. According to DuPont the water uptake in Kapton ${ }^{\circledR}$ is about $4 \% \mathrm{w} / \mathrm{w}$ in $100 \%$ water at $40{ }^{\circ} \mathrm{C}$.

\section{DISCUSSION}

The basics of the DSA technique have been described together with the experimental details and characteristics of the setup. First results on Kapton ${ }^{\circledR}$ films have demonstrated the reproducibility and sensitivity of the DSA technique for the measurement of water vapor absorption in thin polymer films.

Direct comparison of the capacitive response with gravimetric results revealed a linear relationship between the two quantities, which emphasizes the suitability of the DSA technique for the quantitative measurement of mass transport properties of polymer films.

Another feature of the DSA technique is its ability to monitor transport phenomena on a broad time scale varying from seconds to even days (up to saturation). Particularly its fast response time allows the study of highly permeable systems (filters and membranes) as well as the initial stage of diffusion that gives insight in surface properties of coatings or other polymer films.

To exploit the potential of the new technique in the field of nondestructive coating inspection and early warning testing, future work will be dedicated to the investigation of other types of polymer films. In addition, UV degradation will be inflicted on epoxy coatings to determine the sensitivity of the DSA to detect chemical surface changes. Clearly, the DSA technique, in principle, can be used with different gases than the present combination (nitrogen and moisturized nitrogen).

\section{ACKNOWLEDGMENT}

This study is financially supported by SenterNovem IOP-OT.

\section{APPENDIX A}

Talbot solved Eq. (2), without viscoelastic term (thus only the first term, the "perfect" Fickian term), graphically by using the following approximation, putting $\pi^{2} M_{t} / 8 M_{\mathrm{sat}}=x$,

$$
\theta=-\ln x+\frac{q}{p}+\frac{r q^{2}}{2 p^{3}}
$$

where

$$
\begin{aligned}
& \theta=\frac{\pi^{2} D t}{4 l^{2}}, \\
& p=1+x^{8}+x^{24}, \\
& q=\frac{1}{9} x^{8}+\frac{1}{25} x^{24}, \\
& r=1+9 x^{8}+25 x^{24},
\end{aligned}
$$

where $D$ is the diffusion coefficient $\left(\mathrm{m}^{2} / \mathrm{s}\right), t$ is time (s), and $l$ is thickness $(\mathrm{m})$. This graphical solution is correct up to four significant digits when $M_{t} / M_{\text {sat }}<2 /$.

\section{APPENDIX B}

The capacitance of a dry coating is given by

$$
C_{0}=\frac{\varepsilon_{d} \varepsilon_{0} A}{d},
$$

where $C_{0}$ is the capacitance of dry coating $(\mathrm{F}), \varepsilon_{d}$ is the dielectric permittivity of the dry coating, $\varepsilon_{0}$ the permittivity of vacuum $\left(\mathrm{J}^{-1} \mathrm{C}^{2} \mathrm{~m}^{-1}\right), A$ the measured Kapton ${ }^{\circledR}$ film area $\left(\mathrm{m}^{2}\right]$, and $d$ the thickness of the coating $(\mathrm{m})$.

When from time $t=0$ water starts to penetrate from the top of the coating, then there is a wet top layer and a dry bulk layer, which changes in time. The series arrangement of wet and dry layers changing in time is given by

$$
\frac{1}{C_{t}}=\frac{d_{t}}{\varepsilon_{0} \varepsilon_{w} A}+\frac{d-d_{t}}{\varepsilon_{0} \varepsilon_{d} A},
$$

where $C_{t}$ is the resultant capacitance of the coating $(\mathrm{F}), \varepsilon_{w}$ is the dielectric permittivity of the wet coating, and $d_{t}$ the thickness of the wet part of the coating [m].

Because we are interested in the difference of capacitance, just like the difference in mass for gravimetric measurements, we can subtract (B2) from (B1).

$$
\frac{1}{C_{t}}-\frac{1}{C_{0}}=\frac{d_{t}}{\varepsilon_{0} A}\left(\frac{1}{\varepsilon_{w}}-\frac{1}{\varepsilon_{d}}\right) \Rightarrow 1-\frac{C_{0}}{C_{t}}=\frac{d_{t}}{d}\left(1-\frac{\varepsilon_{w}}{\varepsilon_{d}}\right) .
$$

If we assume a simplified diffusion equation,

$$
d_{t}=\sqrt{D t},
$$

where $D$ is the diffusion coefficient $\left(\mathrm{m}^{2} / \mathrm{s}\right)$, and $t$ is time (s).

Replacing $d_{t}$ by Eq. (B4) the resulting equation then becomes

$$
1-\frac{C_{0}}{C_{t}}=\frac{\sqrt{D t}}{d}\left(1-\frac{\varepsilon_{w}}{\varepsilon_{d}}\right) .
$$

Equation (B5) shows that the change of capacitance, due to water ingress, is basically diffusion related and therefore the assumption can be made that $\Delta M_{t}$ can be substituted by $\Delta \varepsilon_{t}^{\prime}$, and $\Delta M_{\text {sat }}$ by $\Delta \varepsilon_{\text {sat }}^{\prime}$

${ }^{1}$ O. G. Piringer and A. L. Baner, Plastic Packaging Materials for Food: Barrier Function, Mass Transport, Quality Assurance, and Legislation (Wiley, New York, 2000).

${ }^{2}$ J. W. Chin, T. Nguyen, and K. Aouadi, J. Appl. Polym. Sci. 71, 483 (1999).

${ }^{3}$ P. Marcus and J. Oudar, Corrosion Mechanisms in Theory and Practice (Dekker, New York, 1995), p. 581.

${ }^{4}$ J. Crank and G. S. Park, Diffusion in Polymers (Academic, New York, 1968), p. 260

${ }^{5}$ J. Comijn, Polymer Permeability (Elsevier, New York, 1985), p. 11.

${ }^{6}$ J. F. Rabek, Experimental Methods in Polymer Chemistry (Wiley, New York, 1980), p. 601.

${ }^{7}$ M. F. García-Sánchez, J. M'Peko, A. R. Ruiz-Salvador, G. Rodríguez-Gattorno, and Y. Echevarría, J. Chem. Educ. 80, 1062 (2003).

${ }^{8}$ R. Buchner, J. Barthel, and J. Stauber, Chem. Phys. Lett. 30, 57 (1999).

${ }^{9}$ M. Heim, R. Eschrich, A. Hillebrand, H. F. Knapp, and R. Guckenberger, J. Vac. Sci. Technol. B 14, 1498 (1996).

${ }^{10}$ G. Wypych, Handbook of Solvents (Chemtec, New York, 2001), p. 305.

${ }^{11} \mathrm{~J}$. Crank, The Mathematics of Diffusion (Clarendon, Oxford, 1975), p. 44.

${ }^{12}$ Y. Sun, Polymer 37, 3921 (1996).

${ }^{13}$ K.-I. Okamoto, N. Tanihara, H. Watanabe, K. Tanaka, H. Kita, A. Nakamura, Y. Kusuki, and K. Nakagawa, J. Polym. Sci., Part B: Polym. Phys. 30, 1223 (1992). 
${ }^{14}$ P. A. M. Steemann and J. van Turnhout, in Broadband Dielectric Spectroscopy (Springer, Heidelberg, 2003), p. 495.

${ }^{15}$ L. D. Landau and E. M. Lifshitz, Electrodynamics of Continuous Media (Pergamon, New York, 1960), p. 46.

${ }^{16}$ H. Looyenga, Physica (Amsterdam) 31, 401 (1965).
${ }^{17}$ A. Talbot and J. A. Kitchener, Br. J. Appl. Phys. 7, 96 (1956).

${ }^{18}$ S. A. Lindqvist, National Association of Corrosion Engineers 41, 69 (1985).

${ }^{19}$ D. Hayward, E. Hollins, P. Johncock, I. McEwan, R. A. Pethrick, and E. A. Pollock, Polymer 38, 1151 (1997). 